\title{
The Epidemiological Characteristics of Pulmonary Tuberculosis - Kashgar Prefecture, Xinjiang Uygur Autonomous Region, China, 2011-2020
}

\author{
Diermulati Tusun'; Maiweilanjiang Abulimiti ${ }^{1}$; Xirizhati Mamuti ${ }^{1}$; Zhenjiang Liu'; Da Xü ${ }^{3}$; Guanzhen Li $^{2}$; \\ Xiaowang Peng'; Tuerhong Abudureyimu'; Lijie Zhang'; Yanlin Zhao ${ }^{3}$; Xichao $\mathrm{Ou}^{3, *}$
}

\section{ABSTRACT}

Introduction: National Notifiable Disease Reporting System (NNDRS) plays an important role in the early detection and control of tuberculosis (TB) in China. This study analyzed the epidemiological characteristics of pulmonary tuberculosis (PTB) in Kashgar Prefecture, Xinjiang Uygur Autnomous Region, China from 2011 to 2020 to provide a scientific basis for developing TB control strategies and measures in Kashgar.

Methods: The data were collected from the NNDRS, which included the geographical distribution, age, sex, occupation, and pathogenic classification of reported PTB cases in 12 counties/cities of Kashgar Prefecture from 2011 to 2020. Descriptive statistics were used to describe the characteristic of PTB epidemic in Kashgar.

Results: There were 189,416 PTB cases reported during 2011-2020, with a mean annual PTB case notification rate (CNR) of 451.29/100,000. A rising trend in the rate of reported PTB between 2011 and $2017\left(\chi_{\text {trend }}^{2}=26.09, P<0.01\right)$ and a declining trend between 2018 and $2020\left(\chi_{\text {trend }}^{2}=314.44, P<0.01\right)$ were observed. The months with the highest reported number of PTB cases were March to May and November to December. The mean annual rate of reported PTB was 451.88/100,000 for males and $450.67 / 100,000$ for females. In addition, $19.76 \%$ of patients were bacteriologically-confirmed (Bac+) cases $(37,425 / 189,416)$, and the mean annual Bac+ CNR was $89.17 / 100,000$, rising from $64.76 / 100,000$ in 2011 to $139.12 / 100,000$ in 2020 ( $\chi_{\text {trend }}^{2}=74.44$, $P<0.01)$.

Conclusions: The CNR of reported PTB in Kashgar showed a significant declining trend in the past three years. Males, elderly population, winter and spring, and farmers as an occupation were the main factors associated with high incidence of tuberculosis in Kashgar. Targeted prevention and treatment of $\mathrm{TB}$ should be strengthened in key groups in this region.

\section{INTRODUCTION}

Tuberculosis (TB) is a chronic respiratory infectious disease caused by Mycobacterium tuberculosis (MTB) infection (1), which can be airborne and poses a serious threat to human health. MTB can invade all organs of the human body but mainly affects the lungs with a typical manifestation of pulmonary tuberculosis (PTB) (2). TB is one of the major infectious diseases affecting the health of public in both China and around the world. The TB epidemic in China is still severe. China is one of the 30 high TB burden countries and ranks second for the number of new estimated TB cases after India $(1,3)$. In 2019, the number of new $\mathrm{TB}$ cases worldwide was about 9.96 million, with TB incidence of 130/100,000 (4). A total of 775,764 PTB cases were reported in the National Notifiable Disease Reporting System (NNDRS) in China, and the rate of reported PTB was 55.6/100,000, ranking second among Class A and Class B notifiable communicable diseases in the country (5). In 2020, the reported TB incidence in Kashgar, a prefecture in Xinjiang Uygur Autonomous Region with 12 counties/cities, was 250.4/100,000, which was 4.3 times that of the national average for the same time period. TB remained one of the major public health concerns in Kashgar. This study reviewed and analyzed the epidemiological characteristics of TB in Kashgar in the past decade to provide scientific evidence to inform the development of TB prevention and control strategies in Kashgar.

\section{METHODS}

The information on geographic distribution, age, sex, occupation, and pathogenic classification of TB patients reported in Kashgar Prefecture during 2011-2020 was collected from the National Notifiable Disease Reporting System (NNDRS), and the 
demographic data were obtained from Kashgar Prefecture Bureau of Statistics.

We processed the data using Microsoft Excel (version 2016, Microsoft, USA). The data were analyzed using SPSS 22.0 (IBM, Chicago, IL, USA). The trend chi-squared test or chi-squared test was conducted to compare the rate of reported PTB in groups with different characteristics, and $P<0.05$ was considered statistically significant.

\section{RESULTS}

In 2011-2020, a total of 189,416 cases of PTB were reported in Kashgar, with the rate of reported cases increased from $402.18 / 100,000$ in 2011 to $524.64 / 100,000$ in $2017\left(\chi_{\text {trend }}^{2}=26.09, P<0.01\right)$ and remarkably declined from $806.75 / 100,000$ in 2018 to $250.74 / 100,000$ in $2020\left(\chi_{\text {trend }}^{2}=314.44, P<0.01\right)$. The mean annual rate of reported PTB from 2011 to 2020 was $450.91 / 100,000$, and the mean annual rate of reported bacteriological confirmed (Bac+) PTB was $89.17 / 100,000$. The rate of reported Bac+ PTB increased from $64.76 / 100,000$ in 2011 to $139.12 / 100,000$ in $2020\left(\chi_{\text {trend }}^{2}=74.44, P<0.01\right)$. The Bac+ cases accounted for $19.76 \%(37,425 / 5.48 \%$ $(6,336 / 11,420)$ in 2020 (Figure 1).

During 2011-2017, there were mainly 2 peaks in the reported cases of PTBs observed in December and in May of each year, accounting for $10.13 \%(19,632 /$ $189,416)$ and $10 \%(19,374 / 189,416)$, respectively. The lowest reported PTB cases was reported in September, accounting for $6.52 \%$ of all reported PTB cases $(12,638 / 189,416)$, and the highest reported PTB cases occurred in the second half of 2018, June of 2019, and May of 2020 (Figure 2).

In 2011-2020, PTB cases were reported in all age groups, with the group of 50-79 years of age accounting for $70.95 \%(134,399 / 189,416)$, but the age group only accounted for $15.46 \%(6,490,776 /$ $41,972,493)$ of the whole population. The top 3 age groups with the highest rate of reported PTB were 75-79 (402.18/100,000), 85+ (4,864.42/100,000), and 70-74 (4,219.59/100,000) (Table 1).

During 2011-2020, the mean annual rate of reported PTB of males was $451.88 / 100,000$, and that of females was $450.67 / 100,000$. Among all patients, males accounted for $50.13 \%(95,123 / 189,416)$ and females accounted for $49.87 \%$ (94,289/189,416). However, as the percentage of male patients increased over the years, there was a statistically significant difference in the ratio of male and female patients $\left(\chi_{\text {trend }}^{2}=204.85, P<0.01\right)$ (Table 2).

In 2011-2020, the top 3 occupations of PTB patients reported were farmers $(83.81 \%, 158,741 /$ 189,416), housework \& unemployed $(6.54 \%$, $12,383 / 189,416)$, and retirees $(4.51 \%, 8,544 /$

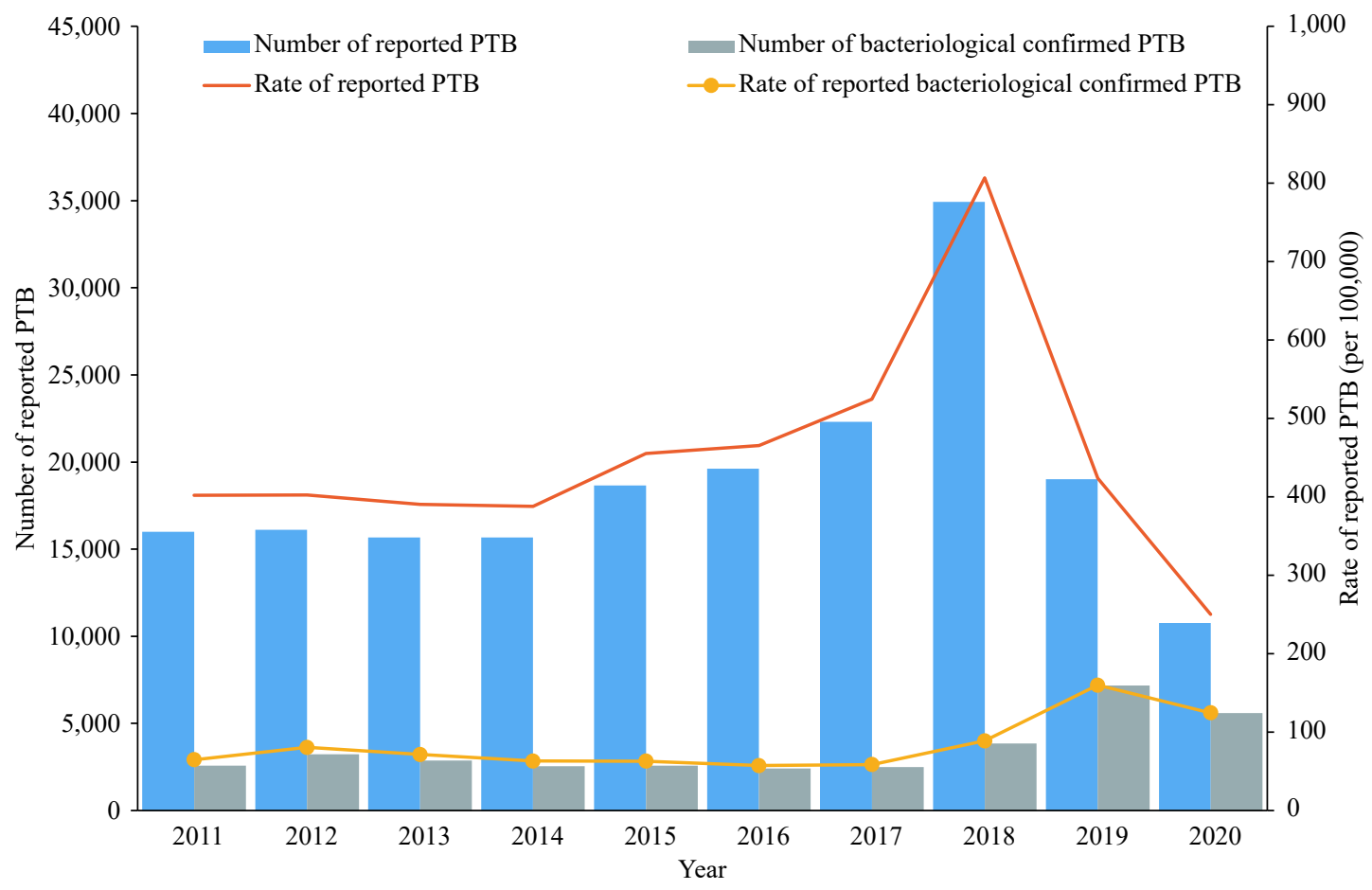

FIGURE 1. The number and case notification rate of reported pulmonary tuberculosis (PTB) in Kashgar, 2011-2020. 


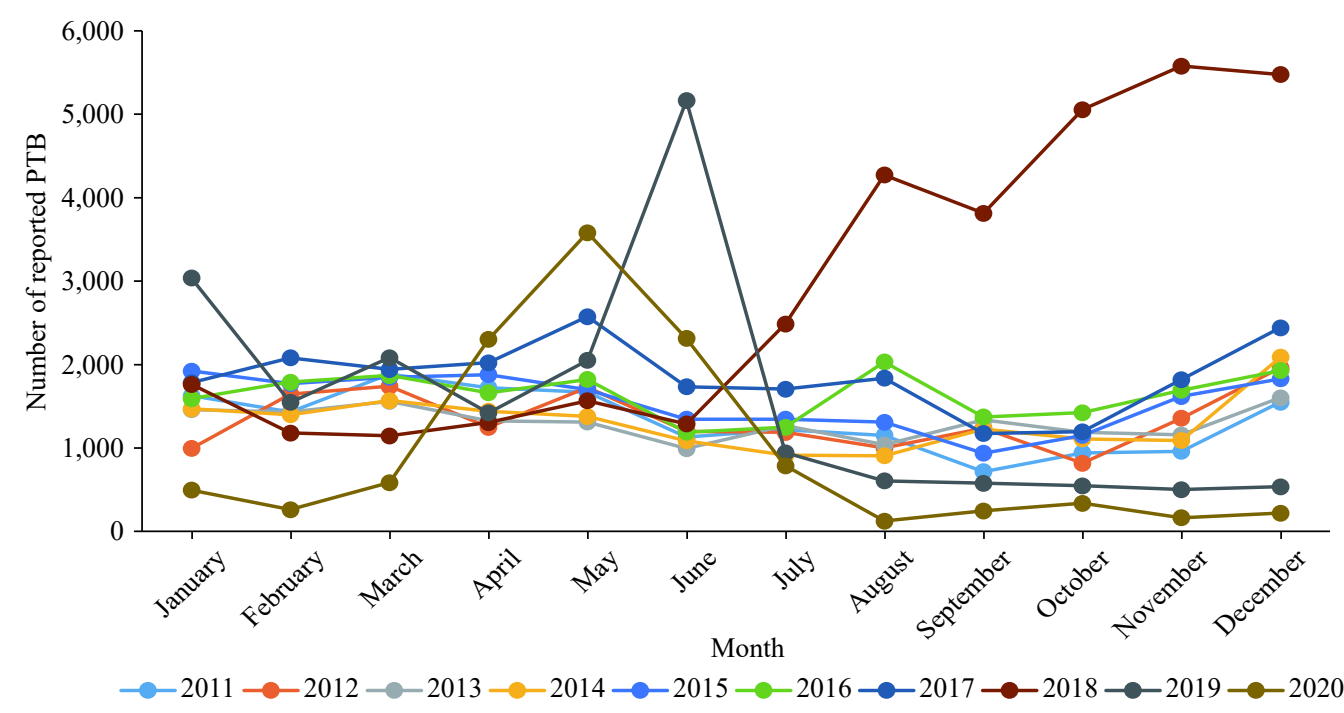

FIGURE 2. Monthly distribution of reported pulmonary tuberculosis (PTB) cases in Kashgar, 2011-2020.

TABLE 1. Age distribution of reported pulmonary tuberculosis cases in Kashgar, 2011-2020.

\begin{tabular}{|c|c|c|c|}
\hline Age (years) & $\begin{array}{l}\text { Number of } \\
\text { reported } \\
\text { cases (n) }\end{array}$ & $\begin{array}{c}\text { Total } \\
\text { population }\end{array}$ & $\begin{array}{c}\text { Average reported } \\
\text { incidence }(1 / 100,000)\end{array}$ \\
\hline $0-4$ & 207 & $4,198,100$ & 4.93 \\
\hline $5-9$ & 161 & $4,021,820$ & 4.00 \\
\hline $10-14$ & 619 & $3,204,956$ & 19.31 \\
\hline $15-19$ & 3,265 & $3,725,311$ & 87.64 \\
\hline $20-24$ & 5,293 & $4,5641,71$ & 115.97 \\
\hline $25-29$ & 6,097 & $4,132,047$ & 147.55 \\
\hline $30-34$ & 5,755 & $3,249,461$ & 177.11 \\
\hline $35-39$ & 5,624 & $3,104,012$ & 181.18 \\
\hline $40-44$ & 6,590 & $2,706,171$ & 243.52 \\
\hline $45-49$ & 8,918 & $2,299,515$ & 387.82 \\
\hline $50-54$ & 12,244 & $1,740,359$ & 703.53 \\
\hline $55-59$ & 18,392 & $1,435,042$ & $1,281.63$ \\
\hline $60-64$ & 27,393 & $1,381,596$ & $1,982.71$ \\
\hline $65-69$ & 32,820 & 963,946 & $3,404.76$ \\
\hline $70-74$ & 26,498 & 627,976 & $4,219.59$ \\
\hline $75-79$ & 17,052 & 341,857 & $4,988.05$ \\
\hline $80-84$ & 7,910 & 182,041 & $4,345.17$ \\
\hline $85+$ & 4,578 & 94,112 & $4,864.42$ \\
\hline $\begin{array}{c}\text { Total } \\
\text { (average) }\end{array}$ & 189,416 & $41,972,493$ & 451.29 \\
\hline
\end{tabular}

$189,416)$, which accounted for $94.86 \%$ of all cases $(179,67 / 189,416)$. The reported average annual PTB rates in Kashgar Prefecture were from Yengisar County (828.09/100,000), Zepu County (656.56/100,000), Yopurga County (556.22/100,000), Shule County (505.57/100,000), Yecheng County $(477.00 / 100,000)$,
Shache County (447.24/100,000), Shuhu County (396.88/100,000), Makit County (377.93/100,000), Bachu County (349.90/100,000), Kashgar City (341.33/100,000), Payzawat County (339.82/ 100,000), and Tashkurghan County (126.68/ 100,000). The 4 counties in southern Kashgar (Yecheng, Zepu, Shache, and Yengisar) accounted for $50.33 \%$ of all cases $(95,335 / 189,416)$.

\section{DISCUSSION}

The results showed that the rate of reported PTB in Kashgar increased from $402.18 / 100,000$ in 2011 to $524.64 / 100,000$ in 2017 and declined from $806.75 / 100,000$ in 2018 to $250.74 / 100,000$ in 2020 , with a significant downward trend in the last three years, declining to the lowest level in 2020. This is mainly due to the launch of TB screening as part of universal health check-up in Kashgar in 2018, in which TB screening test and treatment were provided to people over 15 years old, and about 2700,000 persons were screened each year. The early detection of large numbers of $\mathrm{TB}$ patients has been the primary cause for the significant rise in the CNR of PTB in 2018, and the secondary cause was the launch of the "New TB Prevention and Treatment Service System". Since the launch of the system, referrals within the designated hospitals enhanced patient compliance, reduced patient loss to follow-up, and ensured timely reporting of PTB cases. Meanwhile, Kashgar has implemented a policy of $100 \%$ reimbursement of outpatient care cost for suspected TB patients and over $90 \%$ reimbursement of inpatient care cost for TB patients from 2018, which 
TABLE 2. Gender distribution of reported pulmonary tuberculosis cases in Kashgar, 2011-2020.

\begin{tabular}{cccccccc}
\hline \multirow{2}{*}{ Year } & \multicolumn{3}{c}{ Male } & & \multicolumn{3}{c}{ Female } \\
\cline { 2 - 5 } \cline { 6 - 8 } & Number of cases & Population & Incidence (1/100,000) & & Number of cases & Population & Incidence (1/100,000) \\
\hline 2011 & 7,929 & $2,034,894$ & 389.65 & & 8,073 & $1,944,510$ & 415.17 \\
2012 & 7,800 & $2,005,603$ & 388.91 & & 8,311 & $1,998,390$ & 415.88 \\
2013 & 7,717 & $2,010,057$ & 383.92 & & 7,955 & $2,002,272$ & 397.30 \\
2014 & 7,525 & $2,036,026$ & 369.59 & & 8,153 & $2,003,363$ & 406.97 \\
2015 & 8,884 & $2,053,422$ & 432.64 & & 9,777 & $2,044,954$ & 478.10 \\
2016 & 9,522 & $2,133,707$ & 446.27 & & 10,098 & $2,080,940$ & 485.26 \\
2017 & 11,180 & $2,121,851$ & 526.90 & & 11,126 & $2,129,834$ & 522.39 \\
2018 & 17,981 & $2,149,467$ & 836.53 & & 16,950 & $2,180,376$ & 777.39 \\
2019 & 10,539 & $2,234,589$ & 471.63 & & 8,476 & $2,253,754$ & 376.08 \\
2020 & 6,046 & $2,270,730$ & 266.26 & 5,370 & $2,283,754$ & 235.14 \\
Total & 95,123 & $21,050,346$ & 451.88 & & 94,289 & $20,922,147$ & 450.67 \\
\hline
\end{tabular}

has raised the awareness of voluntary health care seeking of the public.

This study found that the proportion of Bac+ PTB cases in Kashgar increased from $16.10 \%(2,577)$ in 2011 to $55.48 \%(6,336)$ in 2020. The New TB Prevention and Treatment Service System was launched in 2013. In the early stage of the system operation, the quality of sputum collection and laboratory testing declined due to short of $\mathrm{TB}$ professionals at designated hospitals and lack of technical capacity. Thus, a downward trend in the rate of reported Bact patients was observed. Since 2018, the proportion of Bac+ PTB cases has increased significantly, which was related to the combination of smear, culture and molecular biological testing for $\mathrm{TB}$ suspects detected by universal health checkups, as well as the improvement in diagnosis ( 6 ).

In terms of temporal distribution, the highest number of PTB cases were reported in December and January each year, suggesting that winter was the peak season for PTB in Kashgar, which was similar to the findings of other studies (7-8). Possible causes for this included increased chances of survival and transmission of MTB in rural Kashgar during winter when coal was burned for heating, doors and windows were closed, rooms were crowded, and heatable brick beds were carpeted. On the other hand, autumn and winter were the slack farming seasons, in which the possibility of visiting the clinic would increase if people got sick (9). The highest reported PTB cases occurred in the second half of 2018, June of 2019, and May of 2020, which was coincident with the launch of the local TB control policies and the implementation $\mathrm{TB}$ screening as part of the local universal health checkup program launched at different month.
In terms of gender distribution, the number of male patients versus that of female patients increased over the years in Kashgar during 2011-2020. Possible causes included that as men in rural areas had more opportunities to go out to work, they became more mobile and easily to get infected. Some of their behaviors such as smoking and drinking could increase the chances of TB infection. In terms of age, the rate of reported PTB remained steady for patients aged 15-50 years, with a rising trend starting from 50 years of age, peaking at around 65 years of age, then declining rapidly. The peak in men was significantly higher than that in women at around 75 years of age. Overall, the distribution trend was similar to that of other prefectures of Xinjiang Uygur Autonomous Region and other provincial-level administrative divisions (10). The rise in the rate of reported PTB in the age groups above 50 years of age was similar to that of the whole Xinjiang Uygur Autonomous Region (11). The elderly population had lower immunity and was prone to comorbidity with other underlying diseases, making them a high-risk group for the reactivation of latent $\mathrm{TB}$ and development of new TB. In addition, the symptoms of TB in this group were usually atypical and could be easily ignored in diagnosis or misdiagnosed (12). Therefore, symptom monitoring, active screening, and other approaches should be leveraged to increase the early detection of TB for the elderly people (13). In terms of occupation distribution, the majority of $\mathrm{TB}$ patients in Kashgar were farmers, which was similar to that of other parts of China and other prefectures in Xinjiang. The TB incidence was closely related to economic level. Lowincome groups were at higher risk and, together with the general low education level and low health literacy, 
led to less access to TB services because of rural location, longer working hours, and therefore more susceptibility to TB infection (9).

In terms of geographic distribution, the ranking of each county/city for the rate of reported PTB varied each year. It was worth noting that the 4 southern counties of Kashgar accounted for $50.33 \%$ of the total number of reported cases with the highest rate of reported PTB in the prefecture. The serious TB epidemic affecting these 4 counties was related to an underdeveloped economy that mainly depended on agriculture and animal husbandry, the poor living conditions, and the poor health awareness of the residents. Therefore, TB response in these 4 counties should be strengthened.

This study systematically analyzed the epidemiological characteristics of the reported PTB cases in Kashgar from 2011 to 2020, and provided a basis for TB control and prevention. At the same time, there are certain limitations in terms of time distribution and regional distribution, this study only analyzed the overall characteristics of the cumulative number of PTB cases in the past 10 years, further research is needed to reveal the temporal and spatial characteristics of the incidence of PTB in Kashgar. In summary, the continuous improvement of new TB service systems, coupled with the successful implementation of the universal health check-up program, has led to effective control of the TB epidemic in Kashgar, but a large gap is still existed between the rate of reported PTB in Kashgar and the national average in 2020. The next step should focus on the improvement of TB prevention and control in Kashgar, especially the 4 southern counties and the reinforcement of TB case detection. On the basis of routine prevention and control of COVID-19 epidemic, continued efforts should be made to screen key populations for $\mathrm{TB}$ and promote the TB screening as part of the universal health check-up and treatment program, which strives for early detection, diagnosis, and treatment of TB patients (14). Continued efforts should also be made to promote the application of new diagnostic techniques, increase education about and awareness of changing in unhealthy lifestyles, and reinforce the universal screening and treatment of TB, especially among the elderly people, men, and farmers, to further control the TB epidemic in Kashgar, strive to achieve the goals set in the Action Plan to Stop Tuberculosis (2019-2022) in Kashgar, and reach the relevant targets of the "Healthy China 2030" and "Poverty Alleviation in China".
Acknowledgments: Dr. R.J. Simonds and Dr. Zhijun Li from the United States CDC China Office.

doi: $10.46234 / \mathrm{ccdcw} 2021.144$

\# Corresponding author: Xichao Ou, ouxc@chinacdc.cn.

Tuberculosis Dispensary of Kashgar Prefecture, Kashgar, Xinjiang Uygur Autonomous Region, China; ${ }^{2}$ Shandong Provincial Hospital, Jinan, Shandong, China; ${ }^{3}$ Chinese Center for Disease Control and Prevention, Beijing, China.

Submitted: March 30, 2021; Accepted: June 18, 2021

\section{REFERENCES}

1. Ragonnet R, Trauer JM, Geard N, Scott N, McBryde ES. Profiling Mycobacterium tuberculosis transmission and the resulting disease burden in the five highest tuberculosis burden countries. BMC Med 2019;17(1):208. http://dx.doi.org/10.1186/s12916-019-1452-0.

2. Pai M, Behr MA, Dowdy D, Dheda K, Divangahi M, Boehme CC, et al. Tuberculosis. Nat Rev Dis Primers 2016;2:16076. http://dx. doi.org/10.1038/nrdp.2016.76.

3. Floyd K, Glaziou P, Zumla A, Raviglione M. The global tuberculosis epidemic and progress in care, prevention, and research: an overview in year 3 of the End TB era. Lancet Respir Med 2018;6(4):299 - 314 http://dx.doi.org/10.1016/S2213-2600(18)30057-2.

4. World Health Organization. Global tuberculosis reports 2020. 2020. https://www.who.int/tb/publications/global_report/en/. [2020-10-14].

5. Chen W, Zhang H, Du X, Li T, Zhao YL. Characteristics and morbidity of the tuberculosis epidemic -China, 2019. China CDC Wkly 2020;2(12):181 - 4. http://dx.doi.org/10.46234/ccdcw2020.048.

6. Seki M, Kim CK, Hayakawa S, Mitarai S. Recent advances in tuberculosis diagnostics in resource-limited settings. Eur J Clin Microbiol Infect Dis 2018;37(8):1405 - 10. http://dx.doi.org/10.1007/ s10096-018-3258-y.

7. Mao Q, Zeng CH, Zheng DC, Yang YH. Analysis on spatial-temporal distribution characteristics of smear positive pulmonary tuberculosis in China, 2004-2015. Int J Infect Dis 2019;80 Suppl 1:S36-44. http://dx.doi.org/10.1016/j.ijid.2019.02.038.

8. Huang L, Li XX, Abe EM, Xu L, Ruan Y, Cao CL, et al. Spatialtemporal analysis of pulmonary tuberculosis in the northeast of the Yunnan province, People's Republic of China. Infect Dis Poverty 2017;6(1):53. http://dx.doi.org/10.1186/s40249-017-0268-4.

9. Tanimoto $T$, Wang N, Yamamoto K, Morita T, Zhao GM. Incidence of active tuberculosis in rural China. Lancet Infect Dis 2018;18(2): 144 - 5. http://dx.doi.org/10.1016/S1473-3099(18)30021-5.

10. Hao YY, Li YH, Wu G, Xue F, Yao LD, Wang BX, et al. Analysis of epidemiological characteristics of tuberculosis in Aksu Prefecture, Xinjiang in 2011-2015. Chin J Antituberculosis 2017;39(10):1141 - 4. (In Chinese).

11. Dong X, Zhao Z, Liu NQ, Wang SL, Cui Y. Analysis of the characteristics of tuberculosis cases detected among elderly people in Xinjiang Uygur Autonomous Region in 2009-2017. Chin J Antituberculosis 2020;42(9):956 - 61. (In Chinese).

12. Cheng J, Sun YN, Zhang CY, Yu YL, Tang LH, Peng H, et al. Incidence and risk factors of tuberculosis among the elderly population in China: a prospective cohort study. Infect Dis Poverty 2020;9:13. http://dx.doi.org/10.1186/s40249-019-0614-9.

13. Cheng J, Wang LX, Zhang H, Xia YY. Diagnostic value of symptom screening for pulmonary tuberculosis in China. PLoS One 2015;10(5):e0127725. http://dx.doi.org/10.1371/journal.pone.0127725.

14. Huang F, Xia YY, Chen H, Ni W, Xin D, Wei C, et al. The impact of the COVID-19 epidemic on tuberculosis control in China. Lancet Reg Health-West Pac 2020;3:100032. http://dx.doi.org/10.1016/j.lanwpc. 2020.100032 . 